\title{
Sprachliches Verhalten der polnischen Germanistikstudierenden am Beispiel vom Sprechakt ,Einladen“"
}

\section{Speech behavior of the Polish students of German Philology on the example of the speech act of ,inviting"}

Agnieszka Wolak

(Kraków)

\begin{abstract}
The aim of the paper is to present the variety of speech behavior of the Polish students of German Philology performing the speech act of inviting. Inviting is a specific speech act, which demands from L2-learners not only the knowledge of the language learned, but also the knowledge of speech behavior specific for the community which uses the language for everyday communication. The awareness of this fact has an influence on the speech behavior of L2-learners, which shows itself in the quality of utterances used.
\end{abstract}

In jedem Gespräch bedienen sich die Kommunikationspartner unterschiedlicher Sprechakte, die die kleinsten Grundeinheiten der sprachlichen Kommunikation sind (Searle 1969/1987: 29) und deren Hauptaufgabe ist es, die Redeabsichten der beteiligten Personen zum Ausdruck zu bringen. (Searle 1969/1987: 37). Ein Beispiel von einer kommunikativen Situation, in der nicht nur die Kenntnis einer Fremdsprache, sondern auch die Kenntnis der sprachlichen Verhaltensweisen, die für eine sich dieser Sprache bedienenden Gemeinschaft typisch sind, über den kommunikativen Erfolg entscheidet, stellt die Situation dar, in der man eine Einladung macht. 
Das Ziel des Artikels ist die sprachlichen Verhaltensweisen der polnischen Germanistikstudierenden beim Vollzug des Sprechaktes Einladen zu präsentieren.

\section{Einladen im Lichte der Sprechakttheorie}

Einladen können wir zu unterschiedlichen Anlässen. Wir laden unsere Bekannten zu einer Feier, zu einem gemeinsamen Ausgehen, zur Hochzeit ein. Wir können auch fremde Personen zu einem offiziellen Mittagsessen oder zu einem Vorstellungsgespräch einladen. Je nachdem welche Voraussetzungen der Kontext uns stellt und welche Verhaltensweise in der gegebenen Situation üblich ist, können wir uns unterschiedlicher sprachlicher Mittel bedienen. Schon Searle (1969/1987) bemerkte, dass die Auswahl der zur Vollziehung von Sprechakten gebrauchten sprachlichen Mittel eine Sache der Konvention sei, die von einer Sprache zur anderen unterschiedlich sein kann. Einen übersprachlichen und allgemeinen Charakter haben dagegen die den Sprechakten zugrunde liegenden Regeln, die erst in der sprachliche Konvention manifestiert und verwirklicht werden (Searle 1969/1987: 57 f). Diese allgemeinen Regeln versuchte Searle in Form von Glückensbedingungen zu formulieren. Er unterschied folgende Bedingungen, die den erfolgreichen Vollzug eines Sprechaktes gewăhrleisten sollen: Bedingung des propositionalen Gehalts, Einleitungsbedingungen, Aufrichtigkeitsbedingung, wesentliche Bedingung (Searle 1969/1987: $77 \mathrm{ff}$.).

Für den Sprechakt Einladen sind diese Bedingungen folgendermaßen zu formulieren:

1. Bedingung des propositionalen Gehalts - die zukünftige Handlung A wird vom Hörer ausgeführt.

2. Einleitungsbedingung - (1) Der Hörer ist imstande A auszuführen, der Sprecher ist überzeugt, dass der Hörer imstande ist, A auszuführen. (2) Es ist sowohl für den Sprecher als auch für den Hörer offensichtlich, dass sich der Hörer beim normalen Verlauf der Ereignisse verpflichten, A auszuführen. 
3. Aufrichtigkeitsbedingung - Der Sprecher will, dass der Hörer A ausführt.

4. Wesentliche Bedingung - es liegt in der Absicht des Sprechers, den Hörer zur Ausführung von A zu bringen ${ }^{1}$.

Diese Bedingungen wurden von Searle für direkte Sprechakte ausgearbeitet. Direkte Sprechakte sind solche, die mit Hilfe von Äußerungen vollzogen werden, in denen der Sprecher seine Intention/ Redeabsicht explizit zum Ausdruck bringt und die unabhängig vom Kontext in jeder kommunikativen Situation vom Hörer gleich zu verstehen sind, zum Beispiel eine Bitte, die durch die Äußerung: Ich bitte um Ihre Aufmerksamkeit vollzogen wird. Neben diesen können sich die Kommunikationspartner in einer kommunikativen Situation auch sog. indirekter Sprechakte bedienen, oder die Redeabsichten mit Hilfe von para- und nonverbalen Mitteln zum Ausdruck bringen. Als indirekte Sprechakte gelten solche Äußerungen, mit deren Hilfe der Sprecher mehr meint als er sagt. In solchen Fällen „stimmt die Bedeutung der Äußerung in mancher Hinsicht nicht mit der Satzbedeutung überein“" (Searle 1980: 127). Die wirkliche Bedeutung der Äußerung kann nur im Kontext der gegebenen kommunikativen Situation erschlossen werden. Sie werden gebraucht um „einen Illokutionsakt anderer Art zu vollziehen" (Searle 1980: 127). Bei indirekten Sprechakten kommt es sehr oft vor, dass entweder nicht alle Glückensbedingungen beachtet werden, oder eine von ihnen in der Äußerung des Sprechers thematisiert wurde.

Der Kontext, in dem die Interaktion stattfindet, ist also ein sehr wesentliches Element, das das sprachliche Verhalten von Kommunikationspartnern sehr stark beeinflussen kann. Er bildet einen Rahmen der Interaktion und legt die Grenzen fest, die bei der Sicherstellung dessen helfen, was als Kommunikation gilt und was nicht mehr dazu gehört (Morreate, Spitzberg, Berge 2007: 79). Der Kontext

Die Glückensbedingungen für Einladen sind den Glückensbedingungen für den Sprechakt Bitte ähnlich (vgl. Searle 1969/1987: 88), wobei beim Einladen zu bemerken ist, dass die erwartete Handlung symbolisch als A dargestellt, zuerst als eine sprachliche Handlung zu verstehen ist, die eine Form der Annahme der Einladung hat, durch die sich der Hörer verpflichtet zum bestimmten Zeitpunkt am vereinbarten Ort zu erscheinen. 
umfasst solche außersprachliche Faktoren wie: ich, du, hier und jetzt (Awdiejew 2004: 61). Diese Faktoren haben Einfluss darauf, wie die Redeabsichten geäußert werden:

- ich und du - beziehen sich auf die Art des Kontakts, der durch die zwischen den Kommunikationspartnern bestehende Relation bestimmt ist, und spiegeltn sich in der Auswahl des Sprachregisters und in der Direktheit der Äußerungen wider.

- hier und jetzt - bestimmen dagegen Ort und Zeit, zu denen die Interaktion stattfindet. Bei diesen Faktoren werden das allgemeine Weltwissen, sowie auch die bisherigen sprachlichen Erfahrungen und die Muster der typischen sprachlichen Verhaltensweisen in bestimmten kommunikativen Situationen aktiviert, die bei der richtigen Interpretation einer Äußerungen, vor allem wenn eine mit ihrer Hilfe geäußerte Redeabsicht mittels eines indirekten Sprechakts vollzogen wurde, von großer Bedeutung sind (Awdiejew 2004: 61).

Der Kontext, so Searle (1969/1987: 45), weist sehr oft eindeutig darauf hin, welcher illokutionäre $\mathrm{Akt}^{2}$ vollzogen wurde. Ferner bestimmt der Kontext die typischen sprachlichen Verhaltensweisen, die eine sich der gegebenen Sprache bedienende Gemeinschaft in Form der Konvention angenommen hat.

Regeln des sprachlichen Verhaltens, die durch die Konvention bestimmt sind, werden uns impliziert infolge der Sozialisation und

2 Der Begriff illokutionārer Akt, auch als Illokution bezeichnet, stammt von Austin (1962/1972). Er definiert den illokutionären Akt als denjenigen, der die Redeabsicht des Sprechers zum Ausdruck bringt. Austin unterscheidet neben der Illokution noch zwei andere Akte, in die eine Äußerung zergliedert wird. Das sind: die Lokution, anders der lokutionaare $\mathrm{Akt}$ genannt, der dadurch vollzogen wird, dass man etwas sagt. Diesen zerteilt er wieder in drei Akte, nämlich den phonetischen, den phatischen und den rhetischen Akt. Der dritte Akt ist die Perlokution, die auch als der perlokutive Akt bezeichnet wird, und betrifft die Wirkung und Folgen einer Äußerung, die der Sprecher beim Hörer hervorbringen will (Austin 1962/1972: 108 ff.). Searle dagegen spricht von Sprechakten und bezeichnet damit eine besondere Art derAkte, die immer bestimmte Folgen nach sich ziehen und die Einstellungen, Gedanken und Taten des Hörers beeinflussen kônnen. (Searle 1969/1987: 38 f.). Er gebraucht auch die Bezeichnung Illokutionsakt (Searle 1980a, 1980b) in Bezug auf die Redeabsicht, die der Sprecher mit einer Äußerung zum Ausdruck bringt. In dieser Bedeutung wird auch im weiteren Teil des Artikels der Begriff des illokutionären Akts gebraucht. 
der Erziehung beigebracht. „Kinder müssen dazu erzogen werden, sich zu bedanken und sich zu entschuldigen" (Cohen 1980: 33) also in bestimmten Situationen sich sprachlich angemessen zu verhalten. Was aber eine Fremdsprache und ihre Benutzung anbelangt, so sollen diese Regeln im Laufe des Lernprozesses angeeignet werden. Deswegen ist es von großer Bedeutung, dass Sprachlernende nicht nur eine Fremdsprache als System von sprachlichen Zeichen und Regeln ihrer Verbindung lernen, sondern auch mit Gemeinsamkeiten und Unterschieden in den sprachlichen Verhaltensweisen zwischen der Muttersprache und der Zielsprache konfrontiert werden, um mit Angehörigen einer anderen Sprachgemeinschaft in deren Sprache erfolgreich kommunizieren zu können.

\section{Fragestellung}

Eine Gruppe von sehr fortgeschrittenen Fremdsprachelernenden stellen die Germanistikstudierenden dar. Bei dieser Gruppe kann angenommen werden, dass sie nicht nur die deutsche Sprache sehr gut kennt, sondern auch mit den für die deutsche Sprachgemeinschaft typischen sprachlichen Verhaltensweisen vertraut ist. Unter dieser Annahme wurde ein Versuch unternommen, die Frage zu beantworten, inwieweit die Studierenden die zur Vollziehung des Sprechaktes Einladen dienenden Schemata in der deutschen Sprache beherrschten. Alle Überlegungen betreffen eine kommunikative Situation, in der die Interaktion zwischen Nichtmuttersprachlern und Muttersprachlern stattfindet.

Der Schwerpunkt der Darstellung liegt dabei darauf, wie die Studierenden diese Redeabsicht in einer vollzogenen Sprechhandlung verwirklicht haben und ob dies als kontextuell angemessen erscheint. Die Fragen, die in diesem Kontext gestellt wurden, lauten:

1. Auf welche Art und Weise vollziehen die Studierenden den erwünschten illokutionären Akt? Wird dieser so vollzogen, dass die Erkennung der Redeabsicht nur aufgrund des Kontextes möglich ist, also indirekt, oder aber wird er ohne den Kontext weiterhin 
mühelos verstanden, weil der Sprecher genau und direkt seine Redeabsicht ausdrückt?

2. Können sie bestimmte illokutionäre Akte dem dargestellten Kontext angemessen anwenden? Wählen die Studierenden die sprachlichen Mittel aus dem entsprechenden Register, je nach den Voraussetzungen der kommunikativen Situation?

3. Welche formalen Mittel der Sprache (Lexik, Grammatik) werden angewendet?

4. Entsprechen die sprachlichen Verhaltensweisen der Germanistikstudierenden der Norm der deutschen Sprache?

Die im weiteren Teil präsentierten empirischen Daten entstammen den Untersuchungen, die die Autorin im Rahmen ihrer Magisterarbeit mit einer Gruppe von Germanistikstudierenden durchgeführt hat. An den Untersuchungen haben 36 Personen, darunter 19 aus dem 5. Studienjahr und 17 aus dem 4. Studienjahr teilgenommen. Bei der Bewertung der Äußerungen wurden nur solche Fehler berücksichtigt, die die Kommunikation wesentlich beeinträchtigen oder die Vollziehung des erwünschten Sprechaktes unmöglich machen, was die Unangemessenheit der Äußerung im dargestellten Kontext verursacht.

Als Grundlage für die durchgeführte Analyse der Äußerungen von Studierenden wurde die Sprechakttheorie von Searle (1969/1987, 1980a, 1980b, 1999) sowie auch die Theorie der Gesprächsmaximen von Grice (1980) angenommen. Die Basis für die Analyse der grammatischen Erscheinungen bildete die beschreibende Grammatik der deutschen Sprache von Helbig und Buscha (2001). Untersucht wurden vor allem die formalen Merkmale des Sprechaktes in Bezug auf die kommunikative Situation, in der sie vollzogen wurden. Die gesammelten Äußerungen wurden als angemessen, kommunikativ (das bedeutet trotz der auftretenden Fehler die Kommunikation nicht beeinträchtigend) oder unangemessen (das bedeutet in den dargestellten Kontext nicht passend) bewertet. 


\section{Analyse der Äußerungen von Studierenden}

Situation: Za tydzień wydajesz przyjęcie urodzinowe. Zaproś swoich znajomych do wspólnego świętowania.

In dieser Situation sollten die Probanden die Rolle des Gastgebers abspielen, der seine Geburtstagsparty macht, und dazu Gäste einlädt. Die einzuladenden Personen sind seine Bekannten, der Kontakt zwischen den Kommunikationspartnern ist inoffiziell, deswegen sollten die Äußerungen im umgangssprachlichen Stil gehalten werden. Der illokutionäre Akt, der in diesem Kontext zu vollziehen war, ist das Einladen.

Die Mehrheit, 26 von 36 Probanden haben den illokutionären Akt des Einladens indirekt vollzogen, indem sie in einer Äußerung unterschiedliche Sprechakte gebrauchten, die gemeinsam als eine Einladung zu interpretieren sind.

Drei Probanden vollzogen den illokutionären Akt des Einladens indirekt durch die Anwendung des hypothetischen Konditionalsatzes, in dem der Sprecher seine Emotionen darstellt, die durch die zukünftige Handlung des Hörers hervorgerufen werden. Der Konditionalsatz wurde durch eine Behauptung eingeleitet, in der die Informationen über die kommenden Ereignisse vermittelt wurden:

$(\mathrm{V}, 12)$ In einer Woche mach' ich meine Geburtstagsparty, es wäre mir sehr angenehm, wenn ihr kommen würdet. ${ }^{3}$

(IV, 1) Nächste Woche mache ich eine Geburtstagsparty. Es würde mich freuen, wenn ihr auch kommt.

$(I V, 4)$ Nächste Woche mache ich eine Geburtstagsfeier. Ich würde mich freuen, wenn du mitmachen würdest.

In diesem Fall erlaubt die situative Inferenz den Hörern die Schlussfolgerung zu ziehen, dass ihre Anwesenheit auf der Party

3 Alle in diesem Teil angeführten Beispiele stellen die Äußerungen der Probanden dar, die während der Untersuchungen erhoben wurden. Sie werden mit Fehlern, die die Studierenden begingen, übernommen, um ihre Authentizität zu bewahren. Die Markierungen der Äußerungen in runden Klammern bedeuten - die römische Zahl: Studienjahr, die arabische Zahl - die einem Proband zugeteilte Testnummer. 
vom Sprecher erwünscht ist und zu seiner guten Laune beitragen wird, was feststellen lässt, dass die obigen Äußerungen als Einladung zu verstehen sind.

Die Einladung wurde auch indirekt mittels der Entscheidungsfrage gemacht, die durch die in der Behauptung vermittelte Information über das kommende Ereignis ergänzt wurde:

(IV, 3) In einer Woche ist mein Geburtstag. Kommt ihr? Wir feiern es zusammen. Es wird super!

In drei Fällen wurde der erwünschte illokutionäre Akt mittels einer Aufforderung vollzogen, wobei zwei Äußerungen durch eine Behauptung eingeleitet wurden, in der die Informationen über die kommenden Ereignisse mitgeteilt wurden:

$(\mathrm{V}, 4)$ Ich mache nächste Woche eine Geburtstagsparty. Kommt doch gegen 19 Uhr bei mir vorbei.

(IV, 2) In einer Woche gibt's 'ne große Party bei mir. Ich werde nämlich wieder um ein Jahr älter. Kommt mal vorbei! Ihr seid herzlich willkommen.

Durch die Hinzufügung des Satzes: Ihr seid herzlich willkommen. wird zusätzlich markiert, dass diese Äußerung als höfliche Einladung zu verstehen ist.

In der dritten dagegen dient die nachgestellte Behauptung zur Begründung der Einladung:

(IV, 17) Kommt zu mir in einer Woche, ich habe Geburtstag und mache eine Party.

Elf Probanden thematisierten in ihren Äußerungen durch die Anwendung des Modalverbs mögen im Konjunktiv Präteritum in der Bedeutung, Lust, Wunsch haben' die Aufrichtigkeitsbedingung für den illokutionären Akt Einladen, wodurch die Höflichkeit der Einladung unterstrichen wurde. 
$(\mathrm{V}, 5)$ Ich möchte euch zu meinem Geburtstagsfest nächste Woche ganz herzlich einladen.

$(\mathrm{V}, 8)$ Ich möchte euch sehr herzlich zu meinem Geburtstag, der nächste stattfindet, einladen.

$(\mathrm{V}, 14)$ Ich möchte euch zu meiner Geburtstagsparty einladen. Sie findet ...... statt.

$(V, 16)$ Ich möchte euch für meine Geburtstagsparty einladen.

$(V, 18)$ Ich möchte euch zu meiner Geburtstagsparty einladen.

(IV, 8) Ich möchte euch zu meiner Geburtstagsparty einladen.

In vier Fällen wurde ein zusätzlicher Satz hinzugefügt, in dem eine Information über den Termin oder über den Anlass erteilt wurde.

$(\mathrm{V}, 6)$ Ich möchte euch alle herzlich zu meiner Geburtstagsfeier einladen. Die findet am nächsten Mittwoch, um $10 \mathrm{Uhr}$ bei mir zu Hause statt.

(V, 7)Nächste Woche werde ich 24 . Ich mache eine Party und möchte euch herzlich einladen.

$(\mathrm{IV}, 5)$ In einer Woche habe ich Geburtstag und mache eine Geburtstagparty. Ich möchte euch zu dieser Party einladen.

(IV, 10) Nächste Woche mache ich eine Party, weil ich Geburtstag habe. Ich möchte euch einladen. Ich hoffe, dass Ihr kommt.

In Äußerung (IV, 10) wurde außer der Einladung auch eine Behauptung gebraucht, die über den Anlass der Einladung informiert. Die Probandin hat auch mit Hilfe von einer performativen Äußerung ich hoffe, dass die Hoffnung ausgedrückt, dass die Einladung angenommen wird.

(V, 1) Ich möchte Euch zu meiner Geburtstagsfeier einladen.

Es findet heute in einer Woche statt. Ich würde mich freuen, wenn Ihr kommen könnt. 
In der Äußerung (V, 1) wurde die vollzogene Einladung durch eine Behauptung ergänzt, in der die Information über das kommende Ereignis vermittelt wurde. In dem hypothetischen Konditionalsatz drückte die Probandin ihre Emotionen aus, die die Annahme der Einladung bei ihr hervorrufen wird.

(V, 13) Ich will euch zu meinem Geburtstag einladen. Ich hoffe, dass ihr kommt.

In der Äußerung (V, 13) wurde der illokutionäre Akt mittels einer Behauptung vollzogen. Durch die Anwendung des Modalverbs wollen zum Ausdruck der Absicht wurde die Aufrichtigkeitsbedingung dieses Aktes thematisiert. Zusätzlich wurde im zweiten Satz die Hoffnung auf die Annahme der Einladung direkt durch die Anwendung der performativen Äußerung ich hoffe, dass ausgedrückt.

Drei Probanden vollzogen den illokutionären Akt der Einladung direkt durch die Anwendung der allgemeinen Zustandsform eingeladen sein, wodurch die Höflichkeit der Einladung unterstrichen wurde:

$(\mathrm{V}, 2)$ In einer Woche mache ich meine Geburtstagsparty. Ihr seid herzlich eingeladen.

(IV, 7) Nächste Woche gibt's bei mir eine Geburtstagsparty, Ihr seid alle eingeladen.

(IV, 12) Hey! Nächste Woche gibt es bei mir eine Geburtstagsparty. Ihr alle seid natürlich eingeladen!

Alle oben besprochenen Beispiele des sprachlichen Verhaltens wurden als angemessen bewertet.

Die untersuchten Studierenden begingen in ihren Äußerungen vor allem lexikalische Fehler. Nur in einigen Fällen bereiten diese Fehler dem Hörer kleinere Schwierigkeiten bei der Interpretation der Äußerung und Erkennung der Redeabsicht. Diese Äußerungen wurden als kommunikativ bewertet.

In drei Fällen taucht ein Interferenzfehler auf, indem die Probanden die polnische Phrase: wydawać przyjęcie versuchten ins Deutsche zu übersetzen. Dabei wurde das Verb falsch gewählt: 
$(\mathrm{V}, 10)$ In einer Woche gebe ich die Geburtstagsparty Kommt ihr mit?

$(\mathrm{V}, 19)$ In einer Woche werfe ich eine Geburtstagsparty, ich lade euch ein.

(IV, 16) Ich gebe eine Geburtstagsparty in der nächsten Woche. Kommt ihr?

Die korrekte Entsprechung der polnischen Phrase ist: eine Party machen.

Die Verwendung von Daraufhin in der Bedeutung , aus diesem Anlass ${ }^{6}$, verursacht, dass diese Äußerung als zu pompös und übertrieben empfunden wird:

(IV, 13) Ich mache ,ne Party nächste Woche. Daraufhin seid ihr eingeladen.

Was die Äußerung (IV, 9) anbelangt, so haben wir hier mit einem typischen Versprecher zu tun. Die zwei während der Produktion einer Äußerung miteinander konkurrierende aktiven Äußerungspläne, die in die Äußerung einflossen, sind: Geburtstag feiern und Geburtstagsparty machen:

(IV, 9) Nächstes Wochenende feiere ich meine Geburtstagsparty. Es wäre schön, wenn ihr auch kommt!

Sieben Äußerungen sind zwar kommunikativ, werden aber in diesem Kontext als zu direkt empfunden:

$(\mathrm{V}, 3)$ Nächste Woche mache ich Geburtstagsparty. Ich lade euch alle herzlich ein.

$(V, 15)$ Ich lade euch zu meiner Geburtstagsparty ein! Die Party findet in einer Woche statt. Kommt ihr?

$(V, 17)$ Ich lade euch zu meiner Geburtstagsparty ein. $(I V, 6)$ Ich lade euch zu meiner Geburtstagsparty ein. Kommt am Freitag um 20 Uhr.

(IV, 15) Ich lade euch herzlich zu meiner Geburtstagsparty ein! 
(IV, 14) Ich lade euch zu meinem Geburtstag ein.

(IV, 18) Ich lade euch zu meiner Geburtstagsparty ein.

Zwei Äußerungen wurden als unangemessen bewertet.

(V, 9) Ich möchte Sie herzlich zu meinem Geburtstag einladen. Die Party ist in einer Woche um 19.00 will ich sie alle bei mir zu Hause sehen!

In dieser Äußerung $(\mathrm{V}, 9)$ wurde das Modalverb wollen in imperativischer Bedeutung verwendet, wodurch indirekt der illokutionäre Akt des Befehls vollzogen wurde. Die auf diese Weise verfasste Äußerung kann vom Hörer als unhöflich empfunden werden.

(V, 11) Nächste Woche habe ich meinen Geburtstag, ich würde euch ganz herzlich zu mir einladen.

Die Anwendung des Konjunktivs Präteritum (Beispiel V, 11), kann bei dem Hörer den Eindruck hervorrufen, dass diese Einladung ,erzwungen ${ }^{\circ}$ ist, das heißt, der Hörer kann diese Äußerung folgendermaßen verstehen: Ehrlich gesagt hatte ich nicht vor dich einzuladen, aber du hast schon erfahren, dass ich diese Party mache, und unsere gemeinsamen Bekannte schon eingeladen sind, deswegen muss ich dich unter solchen Umständen auch einladen, obwohl ich es wirklich nicht will. In dieser Situation steht der Hörer unter Druck, eine so ausgedrückte Einladung abzulehnen.

\section{Schlussfolgerungen}

Die Studierenden verfügen über die Muster, die dem Sprechakt Einladen zugrunde liegen. Diese werden infolge des Erstspracherwerbs herausgebildet, und müssen nur während der Erlernung des Deutschen durch die für diese Sprache spezifischen Besonderheiten ergänzt werden. Diese Besonderheiten ergeben sich aus den kulturellen Bedingungen, die in der Sprache ihre Wiederspiegelung 
finden. Der Grad der für jede Besonderheit bestimmten Ähnlichkeiten oder Unterschiede im Vergleich zur Muttersprache ist bei der erfolgreichen Vollziehung der Sprechakte maßgebend. In den meisten Fällen hatten die Probanden keine Schwierigkeiten, den erwünschten Sprechakt zu vollziehen. Sie machten das auf indirekte Art und Weise unter Berücksichtigung der sprachlichen Norm und kulturellen Besonderheiten. Beispielweise wurde von Probanden neben der Wendung ich möchte euch einladen, die im Polnischen üblich ist, eine sehr höfliche Wendung Ihr seid herzlich eingeladen gebraucht, die im Polnischen nicht vorkommt.

Bei den erteilten Antworten ist aber zu bemerken, dass diese nach dem Natürlichkeitsgrad eingeteilt werden können. Einige Studierenden haben sich in diesen Situationen sprachlich natürlicher verhalten als andere, d.h. ihre Antworten stehen näher denjenigen Antworten, die von Muttersprachlern erteilt werden könnten. Die Probanden halten sich in ihrer Äußerungen nicht so streng an die in der Schule gelernten Mustert des sprachlichen Verhaltens. Sie sind flexibel und können diese Muster entsprechend der kommunikativen Situation gestalten und anwenden. Diese Probanden können besser zwischen Mitteln aus bestimmten Stilebenen unterscheiden und diese angemessen gebrauchen. Die Antworten der Probanden, die nicht so natürlich klingen, machen in vielen Fällen den Eindruck, als ob sie irgendwann auswendig gelernt worden wären. Sie können als Bausteine bezeichnet werden, die die Grundlage der Kommunikation darstellen, aber nicht weiter ergänzt und ausgebaut wurden. Dies kann mit unterschiedlichen Spracherfahrungen und Anwendungsmöglichkeiten der deutschen Sprache verbunden sein.

Leider konnten sich nicht alle Studierenden in diesen Situationen sprachlich angemessen verhalten und den erwünschten Sprechakt erfolgreich vollziehen.

Die meisten fehlerhaften Antworten sind stilistisch unakzeptabel. Sieben von ihnen waren zu direkt. Eine wird wegen der gebrauchten lexikalischen Einheiten als übertrieben empfunden. In drei Äußerungen kommen Interferenzfehler vor, in einer dagegen kann wegen der falsch eingesetzten lexikalischen Einheiten von einem 
Versprecher die Rede sein. In zwei Fällen sind die Antworten der Situation unangemessen.

Die begangenen Fehler haben in den meisten Fällen zur Beeinträchtigung oder aber auch zur Brechung der Kommunikation geführt. Sie haben auch zu kommunikativen Missverständnissen geführt. Ein Grund dafür kann der Mangel an Spracherfahrung sein, das heißt die Studierenden haben zu wenig Kontakte mit Muttersprachlern oder haben sich zuvor nicht in solch einer kommunikativen Situation befunden. Bei solchen Antworten ist zu bemerken, dass die Studierenden dann vor allem aus ihren Erfahrungen aus der Muttersprache schöpfen. Die Übertragung der sprachlichen Erfahrungen und Verhaltensweisen von der Muttersprache in die Fremdsprache ohne Berücksichtigung der kulturbedingten Unterschiede konnte aber den kommunikativen Erfolg nicht gewährleisten.

\section{Bibliographie}

Austin, John Langshaw (1962/1972): Zur Theorie der Sprechakte. (How to do things with words). Deutsche Bearbeitung von Eike von Savigny. Stuttgart: Philipp Reclam Jun.

Awdiejew, Aleksy (2004): Gramatyka interakcji werbalnej. Kraków: Wydawnictwo UJ.

Awdiejew, Aleksy/Habrajska, Grażyna (2004): Wprowadzenie do gramatyki komunikacyjnej. Łask: Oficyna Wydawnicza Leksem.

Cohen, Laurence Jonathan (1980): „Sprechakte”. [w:] Klußmann, Paul (red.): Sprechakttheroie. Ein Reader. Wiesbaden: Akademische Verlagsgesellschaft Athenaion, 9-52.

Helbig, Gerhard/Buscha, Joachim (2001): Deutsche Grammatik. Ein Handbuch für den Ausländerunterricht. Berlin-München-Wien-Zürich-New York, Langenscheid KG.

Grice, H. Paul (1980): „Logik und Gesprächsanalyse“. [w:] Klußmann, Paul (red.): Sprechakttheroie. Ein Reader. Wiesbaden: Akademische Verlagsgesellschaft Athenaion, 109-126.

Morreale, Sherwyn/Spitzberg, Brian/Barge, Kevin (2007): Komunikacja między ludźmi. Motywacja, wiedza i umiejętności. Warszawa: Wydawnictwo Naukowe PWN. 
Searle, John Rogers (1969/1987): Czynności mowy. Rozważania z filozofii języka. Warszawa: Instytut Wydawniczy Pax.

Searle, John Rogers (1980a): „Eine Klassifikation der Illokutionsakte”. [w:] Klußmann, Paul (red.): Sprechakttheroie. Ein Reader. Wiesbaden: Akademische Verlagsgesellschaft Athenaion, 82-108.

Searle, John Rogers (1980b): „Indirekte Sprechakte”. [w:] Klußmann, Paul (red.): Sprechakttheroie. Ein Reader. Wiesbaden: Akademische Verlagsgesellschaft Athenaion, 127-150.

Searle, John Rogers (1999): Jezzyk, umyst, spoleczeństwo. Filozofia i rzeczywistość. Warszawa: Wydawnictwo W.A.B. / Wydawnictwo CiS. 